Bond University

Research Repository

\title{
A Visual Organic Chemistry Reaction: The Synthesis of 4-Amino-3-nitrobenzoic Acid Methyl Ester via Fischer Esterification
}

Kam, Caleb; Levonis, Stephan M; Schweiker, Stephanie S

Published in:

Journal of Chemical Education

DOI:

10.1021/acs.jchemed.9b01168

Licence:

Other

Link to output in Bond University research repository.

Recommended citation(APA):

Kam, C., Levonis, S. M., \& Schweiker, S. S. (2020). A Visual Organic Chemistry Reaction: The Synthesis of 4Amino-3-nitrobenzoic Acid Methyl Ester via Fischer Esterification. Journal of Chemical Education, 97(7), 19972000. https://doi.org/10.1021/acs.jchemed.9b01168

\section{General rights}

Copyright and moral rights for the publications made accessible in the public portal are retained by the authors and/or other copyright owners and it is a condition of accessing publications that users recognise and abide by the legal requirements associated with these rights.

For more information, or if you believe that this document breaches copyright, please contact the Bond University research repository coordinator 


\section{A Visual Organic Chemistry Reaction - the Synthesis of 4- Amino-3-nitrobenzoic acid methyl ester via Fischer Esterification.}

Caleb M. T. Kam, Stephan M. Levonis, Stephanie S. Schweiker*

5 Medicinal Chemistry Group, Faculty of Health Sciences and Medicine, Bond University, Robina QLD 4229

\section{ABSTRACT}

The synthesis of 4-amino-3-nitrobenzoic acid methyl ester is a simple Fischer esterification reaction designed as an experiment for use in an introductory organic chemistry course. The compound was synthesized as a one-pot reaction within 30 mins to 16 hours, with one hour producing a workable yield. The bright yellow solid was purified using liquid-liquid extraction, and the extraction process is monitored by a marked color change. The product was then characterized by ${ }^{1} \mathrm{H} \mathrm{NMR},{ }^{13} \mathrm{C} \mathrm{NMR}$, and thin-layer chromatography.

\section{GRAPHICAL ABSTRACT}

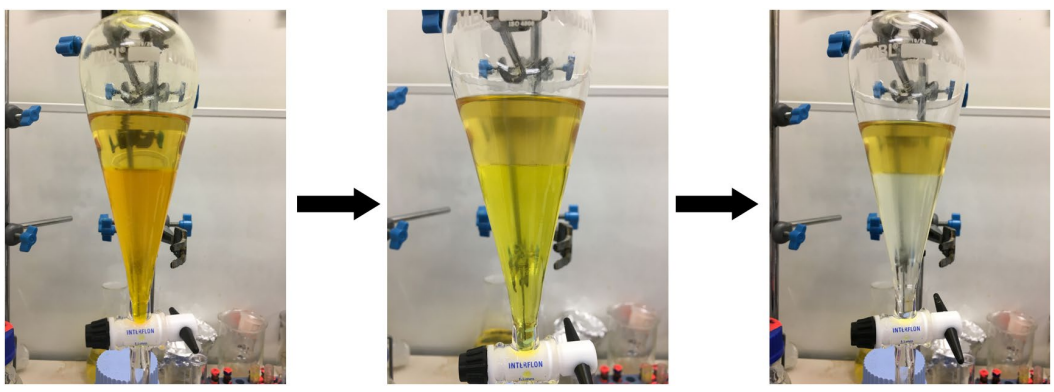

\section{KEYWORDS}

Ester, Synthesis, introductory chemistry, organic chemistry, hands-on learning, visual chemistry.

\section{INTRODUCTION}

Laboratories in undergraduate science incorporate visual, auditory, reading/writing, and kinesthetic aspects. ${ }^{1}$ Fundamental concepts that are difficult to appreciate otherwise, such as solubility and polarity, are demonstrated in laboratory sessions to enhance student learning outcomes. If students are able to visually monitor reactions and purification steps in an organic reaction, it helps to reinforce the main concepts learned. In introductory undergraduate chemistry laboratories, a typical first synthesis experiment would include known reactions such as aspirin synthesis. Aspirin synthesis is a simple and welldocumented reaction with known hazards and risks. The synthesis applies itself to infrared 
spectroscopy with the determination of the added carbonyl group. ${ }^{2}$ However, more commonly,

we see that aspirin synthesis is now often performed in secondary education at high school, and thus there is a drop in the students' engagement if repeated at a tertiary level. ${ }^{3}$

Fischer esterification is an important topic included in most introductory chemistry courses, and examples of these reactions are abundant 4,5 . The reaction is a reversible condensation reaction where a carboxylic acid reacts with an alcohol to produce an ester and water. The amount of water present in the reaction affects the equilibrium position and thus impact on the final yield of the product. Recent research into ester synthesis for undergraduate laboratories focuses on more transferable skills, including isolation of the final product and characterizing using ${ }^{1} \mathrm{H}$ NMR, IR, gas chromatography or thin layer chromatography. ${ }^{6-11}$ This paper proposes the methylation reaction of 4-amino-3-nitrobenzoic acid for use in a typical 2-3 hour laboratory class. The reaction mixture is purified using a liquid-liquid extraction protocol that involves a salt-forming reaction. This extraction process is monitored visually with the carboxylic acid salt moving into the aqueous layer and the final ester retained in the ethyl acetate layer, allowing students to understand the practical approach of using liquid-

45 liquid extraction. Once the extraction is complete, the ethyl acetate layer is filtered and concentrated in vacuo to yield a bright yellow solid. These are the key features that make this experiment a novel example for the teaching of chemistry. This laboratory experiment may be suited to being placed earlier in the curriculum, giving its colorimetric nature and ease, or used in a more advanced educational setting with the application of NMR analysis. 
PEDAGOGICAL GOALS

Fundamental concepts of organic chemistry, such as solubility and polarity, are demonstrated in this experiment. Additionally, the pedagogical goals are:

- To introduce students to experimental organic synthesis techniques found in a traditional research laboratory

55 - To teach and consolidate understanding of the synthesis of ester compounds using Fischer esterification

- To demonstrate to students how to use thin-layer chromatography (TLC)

- To give students an opportunity to perform liquid-liquid extraction and understand the fundamentals of salt formation reactions

60 - To give practical skills to students on how to analyse and characterize organic compounds using IR, NMR, and melting point

- To provide experience to students on how to work safely in an organic chemistry laboratory<smiles>Nc1ccc(C(=O)O)cc1[N+](=O)[O-]</smiles>

1

.<smiles>C1CCCC1</smiles>

conc. $\mathrm{H}_{2} \mathrm{SO}_{4}$
$\mathrm{MeOH}$, reflux

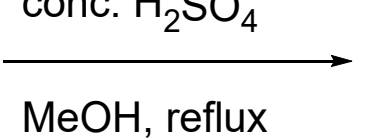

(1)

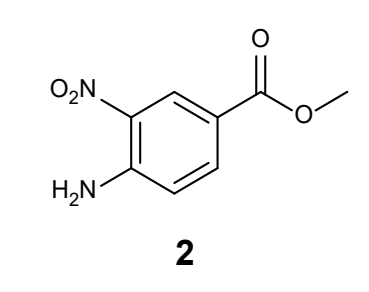

Scheme 1 - Synthesis of compound 2 (4-amino-3-nitrobenzoic acid methyl ester) from compound 1 (4-amino-3-nitrobenzoic acid) in neat methanol with catalytic sulfuric acid.

This document is the Accepted Manuscript version of a Published Work that appeared in final form in Journal of Chemical 
The process was completed in one laboratory period of 3 hours with students working in pairs. The NMR spectra and postlab questions were provided at the end of the laboratory session (see supplementary guides). The first part of this synthesis was the methylation of 4amino-3-nitrobenzoic acid via Fischer esterification using methanol and concentrated

75 sulphuric acid as a catalyst (Scheme 1). Students can monitor and confirm the formation of the product via TLC (with DCM as mobile phase, Figure 1) and visualize under UV light. Since the reaction is heated, in order to check the reaction progression, the reaction was allowed to cool slightly. Students monitored the reaction by TLC at 1 hour from commencement. After TLC, the reaction was quenched with saturated sodium bicarbonate solution and extracted into ethyl acetate, then filtered and dried to yield the final product. The final product's melting point was determined and compared with literature.

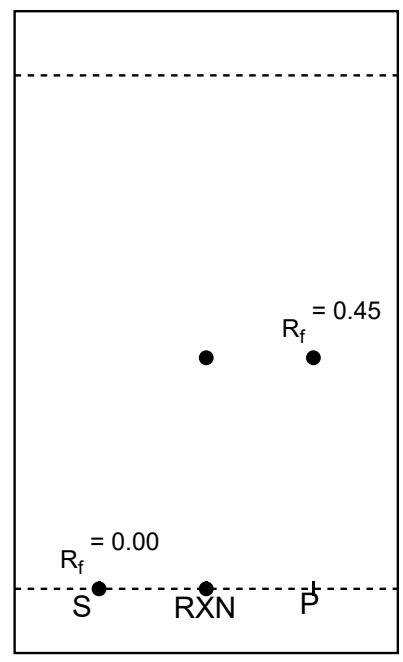

Figure 1: Silica gel thin-layer chromatography (TLC) with fluorescence indicator (f254) for visualization under UV light. S = starting compound (4-amino-3-nitrobenzoic acid), RXN = reaction mixture (monitored during the reaction), and $\mathrm{P}=$ product (4amino-3-nitrobenzoic acid methyl ester).

\section{HAZARDS}

The solvents used in this experiment, methanol and ethyl acetate are highly volatile, flammable, and of moderate toxicity. Dichloromethane is highly volatile, and excessive DCM could cause drowsiness and has moderate toxicity. 4-amino-3-nitrobenzoic acid and 4amino-3-nitrobenzoic acid methyl ester (methyl 4-amino-3-nitrobenzoate) are irritants and may cause skin, eye, and respiratory irritation. Sulphuric acid is corrosive. The synthesis 
step requires the heating of a flammable solvent. The reaction must be conducted in a fume extraction hood, and fire extinguishing equipment must be in place. Organic and aqueous waste should be disposed of correctly. Gloves, lab coat, and appropriate eye protection are required.

\section{RESULTS AND DISCUSSION}

Reaction Time

To establish a reasonable reaction time for an undergraduate laboratory, the reaction mixture was heated at reflux for 30 minutes, 1 hour, 2 hours, and 16 hours (yields detailed in Table 1). A reaction of 1 hour represented reasonable yields for isolation of the final compound and was an appropriate time for the undergraduate laboratory session.

Table 1 Correlation of Reaction Time and Percentage Yield

$\begin{array}{ll}\text { Reaction Elapsed Time } & \text { Percentage Yield } \\ 30 \text { Minutes } & 20 \% \\ 1 \text { Hour } & 46 \% \\ 2 \text { Hours } & 50 \% \\ 16 \text { Hours } & 95 \%\end{array}$

\section{Visual Reaction Workup}

The workup procedure utilizes a salt-forming reaction along with polarity differences, and

110 was visually monitored by the students. The starting material, compound 1, 4-amino-3nitrobenzoic acid, contains a carboxylic acid, which reacts with sodium bicarbonate to form the corresponding sodium salt (shown below, Figure 2), and becomes soluble in the aqueous phase. The product, 4-amino-3-nitrobenzoic acid methyl ester, is not affected by the sodium bicarbonate and remains highly soluble in ethyl acetate and remains into the organic phase during the liquid-liquid extraction.<smiles>Nc1ccc(C(=O)O[Na])cc1[N+](=O)[O-]</smiles><smiles>COC(=O)c1ccc(N)c([N+](=O)[O-])c1</smiles>

Ethyl acetate Soluble 
Figure 2: 4-Amino-3-nitrobenzoic acid sodium salt (right-hand side) and 4-amino-3-nitrobenzoic acid methyl ester (left-hand side).

Once the reaction time was finished, the reaction was cooled, and a solution of saturated sodium bicarbonate was added to quench the reaction. From there, the organic phase was washed with the saturated sodium bicarbonate solution for a total of four times, or until the aqueous phase had become clear (Figure 3). Since the sodium carboxylate is also colored, its removal was monitored in this way. Students were thus able to monitor the purification progress of this reaction visually. Once the ethyl acetate layer was extracted, it was filtered through a silica plug and then concentrated in vacuo to yield the final product as a bright yellow solid.

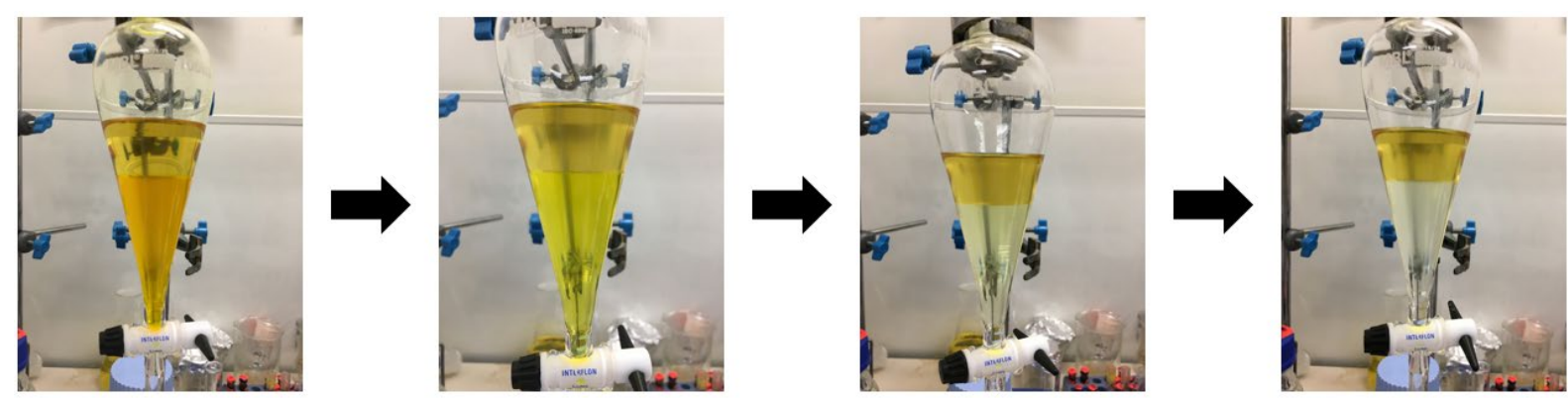

130 Figure 3: Liquid-liquid extraction of 4-amino-3-nitrobenzoic acid methyl ester with ethyl acetate (top layer) and saturated sodium bicarbonate (bottom layer). Demonstrating the disappearing yellow colour of the bottom layer after successive washes with sodium bicarbonate.

\section{Analysis and Elucidation of Structure}

The final methyl ester compound, 4-amino-3-nitrobenzoic acid methyl ester was elucidated by both ${ }^{1} \mathrm{H}$ NMR and ${ }^{13} \mathrm{C}$ NMR, and the melting point determined. The melting point range was determined to be $188-191^{\circ} \mathrm{C}$ for the final product compared to $290-297^{\circ} \mathrm{C}$ for the starting 4-amino-3-nitrobenzoic acid, which is consistent with the literature. ${ }^{12}$ The ${ }^{1} \mathrm{H}$ NMR spectra was provided to the students at the end of the laboratory session and showed

140 five proton environments represented by five peaks. Due to the types of hydrogen environments within the compound, a low field benchtop NMR would be able to distinguishable them and thus elucidate the structure. When comparing the product to the starting material, there is a new singlet peak observed at $3.80 \mathrm{ppm}$ representing the ester 
methoxy $\mathrm{CH}_{3}$ which was introduced in the reactant and not observed in the starting material

145 (Figure 4). The other peaks at 7.04, 7.82, and $8.52 \mathrm{ppm}$ elucidate the benzene ring with the doublet at $7.04(J=8.95 \mathrm{~Hz})$ indicating a meta-proton, the doublet of doublets at $7.82 \mathrm{ppm}$ $(J=2.10,8.95 \mathrm{~Hz})$ indicating an ortho-proton, and the final doublet at $8.52 \mathrm{ppm}(J=2.01$ $\mathrm{Hz}$ ) indicates the remaining proton. The broad peak at $7.97 \mathrm{ppm}$ is indicative of an aromatic amine. On a $400 \mathrm{MHz} \mathrm{NMR}$, the proton $J$ coupling values were determined, which corresponded to the expected values for an ortho- meta- and para- protons.

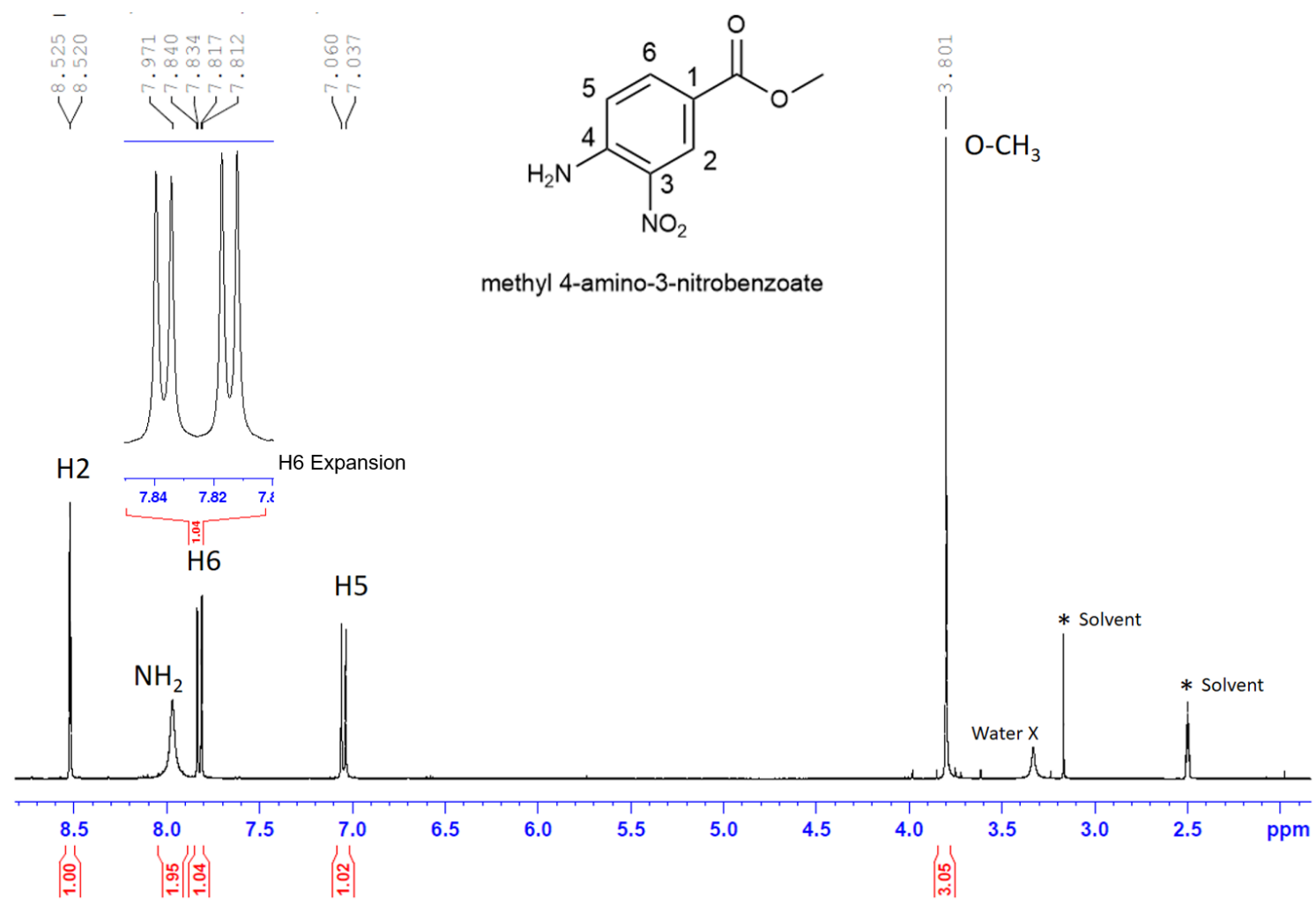

Figure 4: ${ }^{1} \mathrm{H}$ NMR of 4-amino-3-nitrobenzoic acid methyl ester at $400 \mathrm{MHz}$ in DMSO-d6.

The carbon spectrum shows eight carbon peaks which correspond to the methoxy carbon at $51.9 \mathrm{ppm}$, and the aromatic region carbons at $116.1,119.3,128.0,129.6,134.7$, and $148.8 \mathrm{ppm}$. The other significant peak is the carbonyl at $164.9 \mathrm{ppm}$ (instructors notes). 
In conclusion, this reaction is simple and cost-effective as a teaching reaction. The purification, liquid/liquid extraction process, provides a visual opportunity for students to track the salt-forming reaction and consolidate their understanding. Students can assign proton and carbon NMR or IR as well as determine the melting point and compare it with the starting 4-amino-3-nitrobenzoic acid. The experimental outcomes are consistent with existing curricula and offers a unique visual organic chemistry laboratory experience.

\section{ASSOCIATED CONTENT}

Supporting Information

The Supporting Information is available on the ACS Publications website at DOI:

[Instructor notes] including:

1. Chemical materials for experiments: reagent and solvent tables

2. Hazards and Safety Instructions

1753 . Instructor notes

4. Results for post lab questions (including the full assignment for ${ }^{1} \mathrm{H}$ NMR and ${ }^{13} \mathrm{C}$ NMR spectra).

[Student laboratory handout] including:

1. Safety instructions

2. Experimental procedure

3. Postlab questions.

\section{AUTHOR INFORMATION}

185 Corresponding Author

*E-mail: sschweik@bond.edu.au

\section{ACKNOWLEDGMENTS}

A special thanks to the students who undertook the laboratory experiment for the first time and showed that it could be incorporated into our introductory organic chemistry laboratory course.

\section{REFERENCES}


1. Learning strategies and learning styles. Plenum Press: New York, NY, US, 1988; 368.

$1952 . \quad$ Olmsted, J. A., Synthesis of Aspirin: A General Chemistry Experiment. Journal of Chemical Education 1998, 75 (10), 1261.

3. Barry, E.; Borer, L. L., Experiments with Aspirin. J. Chem. Educ. 2000, 77 (3), 354.

4. $\quad$ Yearty, K. L.; Sharp, J. T.; Meehan, E. K.; Wallace, D. R.; Jackson, D. M.; 200 Morrison, R. W., Implementation of picoSpin Benchtop NMR Instruments into Organic Chemistry Teaching Laboratories through Spectral Analysis of Fischer Esterification Products. Journal of Chemical Education 2017, 94 (7), 932-935.

5. Reilly, M. K.; King, R. P.; Wagner, A. J.; King, S. M., Microwave-Assisted Esterification: A Discovery-Based Microscale Laboratory Experiment. Journal of 205 Chemical Education 2014, 91 (10), 1706-1709.

6. Brown, D. P.; Durutlic, H.; Juste, D., Spectroscopic Properties of Some Simple Esters. A Practical Application of Synthesis and Spectroscopy in the Undergraduate Organic Laboratory. J. Chem. Educ. 2004, 81 (7), 1016.

7. Kolar, P.; Tisler, M., A Convenient Synthesis of the Tetrasubstituted Pyrrole: An

210 Undergraduate Heterocyclic Laboratory Experiment. J. Chem. Educ. 1996, 73 (10), 986.

8. Hoogenboom, B. E.; Ihrig, P. J.; Langsjoen, A. N.; Linn, C. J.; Mulder, S. D., The malonic ester synthesis in the undergraduate laboratory. J. Chem. Educ. 1991, 68 (8), 689.

2159 9. Kelly, L. F., A synthesis of chrysanthemic ester: An undergraduate experiment. J. Chem. Educ. 1987, 64 (12), 1061.

10. McCullagh, J. V.; Hirakis, S. P., Synthesis of the Commercial Fragrance Compound Ethyl 6-Acetoxyhexanoate: A Multistep Ester Experiment for the SecondYear Organic Laboratory. J. Chem. Educ. 2017, 94 (9), 1347-1351.

220 11. Sichula, V. A., Synthesis of 10-Ethyl Flavin: A Multistep Synthesis Organic Chemistry Laboratory Experiment for Upper-Division Undergraduate Students. J. Chem. Educ. 2015, 92 (9), 1539-1542.

12. Thomas, P. R.; Tyler, G. J., 421. Some new benziminazole derivatives. J. Chem. Soc. 1957, (0), 2197-2201. 